\title{
Activating transcription factor 3 regulates canonical TGF $\beta$ signalling in systemic sclerosis
}

\author{
Tatjana Mallano, ${ }^{1}$ Katrin Palumbo-Zerr, ${ }^{1}$ Pawel Zerr, ${ }^{1}$ Andreas Ramming, ${ }^{1}$ \\ Barbara Zeller, ${ }_{1}^{1}$ Christian Beyer, ${ }_{1}^{1}$ Clara Dees, ${ }^{1}$ Jingang Huang, ${ }_{1}^{1}$ Tsonwin Hai, $^{2}$ \\ Oliver Distler, ${ }^{3}$ Georg Schett, ${ }^{1}$ Jörg H W Distler ${ }^{1}$
}

\begin{abstract}
Handling editor Tore K Kvien
${ }^{1}$ Department of Internal

Medicine 3, Institute for

Clinical Immunology, University of Erlangen-Nuremberg,

Erlangen, Germany

${ }^{2}$ Department of Molecular and

Cellular Biochemistry, The Ohio

State University, Columbus,

Ohio, USA

${ }^{3}$ Rheumaklinik, University

Hospital Zurich, Zurich,

Switzerland
\end{abstract}

\section{Correspondence to}

Dr Jörg H W Distler,

Department of Medicine 3,

Institute for Clinical

Immunology, University of

Erlangen-Nuremberg,

Ulmenweg 18, D-91054

Erlangen, Germany;

Joerg.distler@uk-erlangen.de

Received 4 July 2014

Revised 4 November 2014

Accepted 20 December 2014

Published Online First

14 January 2015

\section{ABSTRACT}

Background Activating transcription factor 3 (ATF3), a member of the ATF/cAMP-responsive element binding (CREB) family of transcription factors, regulates cellular response to stress including oxidative stress. The aim of this study was to analyse the role of ATF3 in fibroblast activation in systemic sclerosis (SSC).

Methods ATF3 was analysed by reverse transcription quantitative $P C R$, western blot and immunohistochemistry. ATF3 knockout fibroblasts and mice were used to study the functional role of ATF3. Knockdown experiments, reporter assays and coimmunoprecipitation were performed to study the effects of ATF3 on Smad and activation protein 1 (AP-1) signalling. The role of c-Jun was analysed by costaining, specific inactivation and coimmunoprecipitation. Results Transforming growth factor- $\beta$ (TGF $\beta$ ) upregulates the expression of ATF3 in SSC fibroblasts. ATF3-deficient fibroblasts were less sensitive to TGF $\beta$, whereas ectopic expression of ATF3 enhanced the profibrotic effects of TGF $\beta$. Mechanistically, ATF3 interacts with Smad3 directly on stimulation with TGF $\beta$ and regulates Smad activity in a c-Jun-dependent manner. Knockout of ATF3 protected mice from bleomycin-induced fibrosis and fibrosis induced by overexpression of a constitutively active TGF $\beta$ receptor I. Reporter assays and analyses of the expression of Smad target genes demonstrated that binding of ATF3 regulates the transcriptional activity of Smad3.

Conclusions We demonstrate for the first time a key role for ATF3 in fibrosis. Knockout of the ATF3 gene reduced the stimulatory effect of TGF $\beta$ on fibroblasts by interfering with canonical Smad signalling and protected the mice from experimental fibrosis in two different models. ATF3 might thus be a candidate for molecular targeted therapies for SSC.

\section{INTRODUCTION}

Systemic sclerosis (SSc) is a chronic fibrotic connective tissue disease of unknown aetiology that affects the skin and internal organs. It is characterised by an aberrant activation of fibroblasts, resulting in excessive release of extracellular matrix. ${ }^{1}$ Accumulation of extracellular matrix proteins disturbs the physiological tissue function and causes high morbidity and mortality. Transforming growth factor- $\beta$ (TGF $\beta$ ) has been characterised as a key mediator of fibroblast activation in SSc and other fibrotic diseases. ${ }^{2}$ However, the intracellular signalling cascades that control TGF $\beta$ signalling and the TGF $\beta$-induced activation of fibroblasts are still incompletely understood.

The family of activating transcription factor (ATF) consists of a large group of transcription factor members such as cAMP-responsive element binding protein (CREB), cAMP-response element modulator (CREM), B-ATF and ATF1-7. ${ }^{3}$ All family members can regulate gene expression via binding to consensus ATF/CREB cis-regulatory element via a basic-region leucine zipper domain (bZIP). The leucine zipper region is responsible for forming homodimers or heterodimers with other bZIP-containing proteins, while the basic region in this domain is responsible for specific DNA binding. ${ }^{4}$

Initially, ATF3 was suggested to regulate gene expression on the basis of the size of its isoformsfor example, the longer isoform is known to repress designated genes rather than to activate them. ${ }^{5}$ However, later investigations revealed that ATF3 can also form dimers with other ATF/CREB protein members such as ATF2, ${ }^{6}$ c-Jun, JunB ${ }^{7-9}$ and JunD. ${ }^{10-12}$ Depending on the promoter context, these heterodimers can act as either repressors or activators of transcription. ${ }^{13}$ This suggests a diverse repertoire of ATF3 functions depending on the cellular context and organs involved. Indeed, the expression of ATF3 is kept low in quiescent cells, while it can be rapidly induced by a wide variety of signals of cellular stress such as DNA damage, oxidative stress and cell injury, ${ }^{2} 314$ as well as by several cytokines such as interleukin-1 $\beta$, tumour necrosis factor- $\alpha$ and TGF $\beta{ }^{13}{ }^{15}$ Given the key role of oxidative stress in the pathogenesis of $\mathrm{SSc},{ }^{16}$ we thus aimed to study the role of ATF3 in fibroblast activation in SSc. Here, we demonstrate that TGF $\beta$ stimulates the expression of ATF3 in dermal fibroblasts and that ATF3 contributes to the profibrotic effects of TGF $\beta$ and to the formation of fibrosis in two mouse models. Significantly, ATF3 is elevated in the skin of patients with SSc, suggesting its relevance to human fibrosis.

\section{MATERIALS AND METHODS}

\section{Patients}

Dermal fibroblasts were isolated from skin biopsy specimens from 23 patients with SSc and 21 ageand sex-matched healthy volunteers. All patients fulfilled the American College of Rheumatology/ European League Against Rheumatism criteria from 2013. ${ }^{17}$ Sixteen patients were female, seven were 
male. The median age of patients with SSc was 45 years (range 19-65), and their median disease duration was 5 years (range 0.5-10). All patients and controls signed a consent form approved by the local institutional review board. Patients did not receive any potentially disease-modifying agents at the time of biopsy.

\section{Reverse transcription coupled with quantitative PCR}

Gene expression was quantified by reverse transcription coupled with SYBR Green quantitative PCR (RT-qPCR) using the MX3005P Detection System (Agilent Technologies, Böblingen, Germany). Samples without enzyme in the reverse transcription reaction were used as negative controls. Unspecific signals caused by primer dimers were excluded by non-template controls and by dissociation curve analysis as described ${ }^{18-20} \beta$-Actin was used to normalise for the amounts of cDNA within each sample.

\section{Western blot analysis}

The protein concentration of cell lysates was determined by amido black assays. Proteins were separated by sodium dodecyl sulfate/polyacrylamide gel electrophoresis (SDS-PAGE) and transferred to a poly(vinylidene difluoride) membrane. The membrane was incubated with antibodies against Smad3 (Santa Cruz Technologies, Heidelberg, Germany) or ATF3 (Santa Cruz Technologies) and horseradish peroxidase (HRP)-conjugated secondary antibodies (Dako, Hamburg, Germany). Blots were visualised by enhanced chemiluminescence and quantified by density analysis using ImageJ. $\beta$-Actin served as loading control. $^{2122}$

\section{Quantification of collagen protein}

The amount of soluble collagen in cell culture supernatants was quantified using the SirCol collagen assay (Biocolor, Belfast, Northern Ireland). ${ }^{23}{ }^{24}$ The total collagen content of tissue samples was determined by hydroxyproline assays.

\section{Immunohistochemistry and immunofluorescence staining}

Formalin-fixed, paraffin-embedded skin sections or fibroblasts fixed in $4 \%$ paraformaldehyde and permeabilised with $0.25 \%$ Triton X-100 were stained with antibodies against prolyl-4hydroxylase $\beta$ (Life Technologies), ATF3, vimentin (both Abcam) and c-Jun (Santa Cruz). HRP-conjugated or Alexa Fluor antibodies (Life Technologies) were used as secondary antibodies. Irrelevant isotype matched antibodies served as controls. Nuclei were counterstained using 4',6-diamidino-2-phenylindole (Santa Cruz Biotechnology). Staining was analysed using a Nikon Eclipse 80i microscope (Nikon, Badhoevedorp, The Netherlands).

\section{Coimmunoprecipitation (CoIP)}

Fibroblasts were collected in $150 \mathrm{mM} \mathrm{NaCl}$ lysis buffer. Twenty per cent of the lysates was used as loading control (input). Cell extracts were incubated with $20 \mu \mathrm{L}$ protein $\mathrm{A} / \mathrm{G}$ Sepharose and antibodies against $\mathrm{Smad} 3$, ATF3, c-Jun or IgG control immunoglobulins (Santa Cruz Biotechnology). The amounts of the respective proteins of interest in the immunoprecipitate were analysed by western blotting.

\section{Nucleofection}

Fibroblasts were transfected with $10 \mu \mathrm{g}$ pCMV-ATF3 or pCMV plasmid constructs in the absence or presence of the luciferase reporter driven by the CAGA Smad element using the Amaxa 4D-Nucleofector (Amaxa, Cologne, Germany). ${ }^{25}$ Transfection efficiency was determined by cotransfection with vectors encoding $\beta$-galactosidase (Promega, Mannheim, Germany).

\section{Animal studies}

The role of ATF3 in fibrosis was investigated in two different mouse models. (i) In the model of bleomycin-induced dermal fibrosis, fibrosis was induced by local injections of bleomycin for 4 weeks. Subcutaneous injections of $0.9 \% \mathrm{NaCl}$ served as control. (ii) In the TGF $\beta$ receptor I (TBRI) model, injections of replication-deficient type 5 adenoviruses encoding a constitutively active TBRI construct induced localised skin fibrosis. Mice injected with type 5 adenoviruses encoding LacZ served as controls; $6.67 \times 10^{7}$ infectious units were injected intracutaneously. Fibrotic changes were analysed by quantification of the dermal thickness, myofibroblast counts and the hydroxyproline content as described previously. ${ }^{26}{ }^{27}$ For direct visualisation of collagen, trichrome staining was performed.

\section{Statistical analysis}

All data are presented as mean with SEM, and differences between the groups were tested for their statistical significance by the non-parametric Mann-Whitney $U$ test. p Values $<0.05$ were considered significant.

\section{RESULTS}

\section{Overexpression of ATF3 in SSc fibroblasts}

We found increased expression of ATF3 in the skin of patients with SSc compared with matched healthy individuals (figure 1A). In particular, spindle shaped cells in the dermis stained intensely for ATF3. Costaining with the fibroblast marker, prolyl-4hydroxylase $\beta$, confirmed that SSc fibroblasts express high levels of ATF3 and demonstrated that all SSc fibroblasts stained for ATF3, whereas only a minority of fibroblasts express ATF3 in sections from healthy volunteers (figure 1B). Semiquantitative analysis confirmed that the levels of ATF3 are upregulated in fibroblasts of patients with SSc (figure 1C). Although there was a trend towards higher expression levels in patients with active and diffuse cutaneous SSc, this did not reach statistical significance. The upregulation of ATF3 persisted in SSc fibroblasts even after several passages in culture (figure $1 \mathrm{D}, \mathrm{E}$ ), indicating that an endogenous mechanism may account for the overexpression of ATF3 in SSc fibroblasts.

\section{ATF3 is upregulated in a TGF $\beta$-dependent manner}

Since activation of TGF $\beta$ signalling is a core pathway for fibroblast activation and its persistent activation is a hallmark of SSc fibroblasts, we investigated whether TGF $\beta$ stimulates ATF3 expression. Stimulation of healthy human fibroblasts with recombinant TGF $\beta$ rapidly increased the mRNA levels of ATF3, with a peak as early as $1 \mathrm{~h}$ after stimulation (figure $2 \mathrm{~A}$ ). The protein levels of ATF3 increased progressively within the first $24 \mathrm{~h}$ after stimulation with TGF $\beta$ (figure 2B). Knockdown of Smad3 abrogated the stimulatory effects of TGF $\beta$ on ATF3 (figure 2C, D), demonstrating that TGF $\beta$ induces ATF3 in fibroblasts via the canonical Smad pathway. We further tested the role of TGF $\beta$ in ATF3 expression using a mouse model of bleomycin-induced skin fibrosis. Selective inhibition of TGF $\beta$ signalling by SD208, a TBRI inhibitor, ${ }^{28}$ completely prevented the upregulation of ATF3 in bleomycin-challenged mice (figure 2E, F). Taken together, these results demonstrate that ATF3 is overexpressed in SSc fibroblasts in a TGF $/$ Smad-dependent manner. 

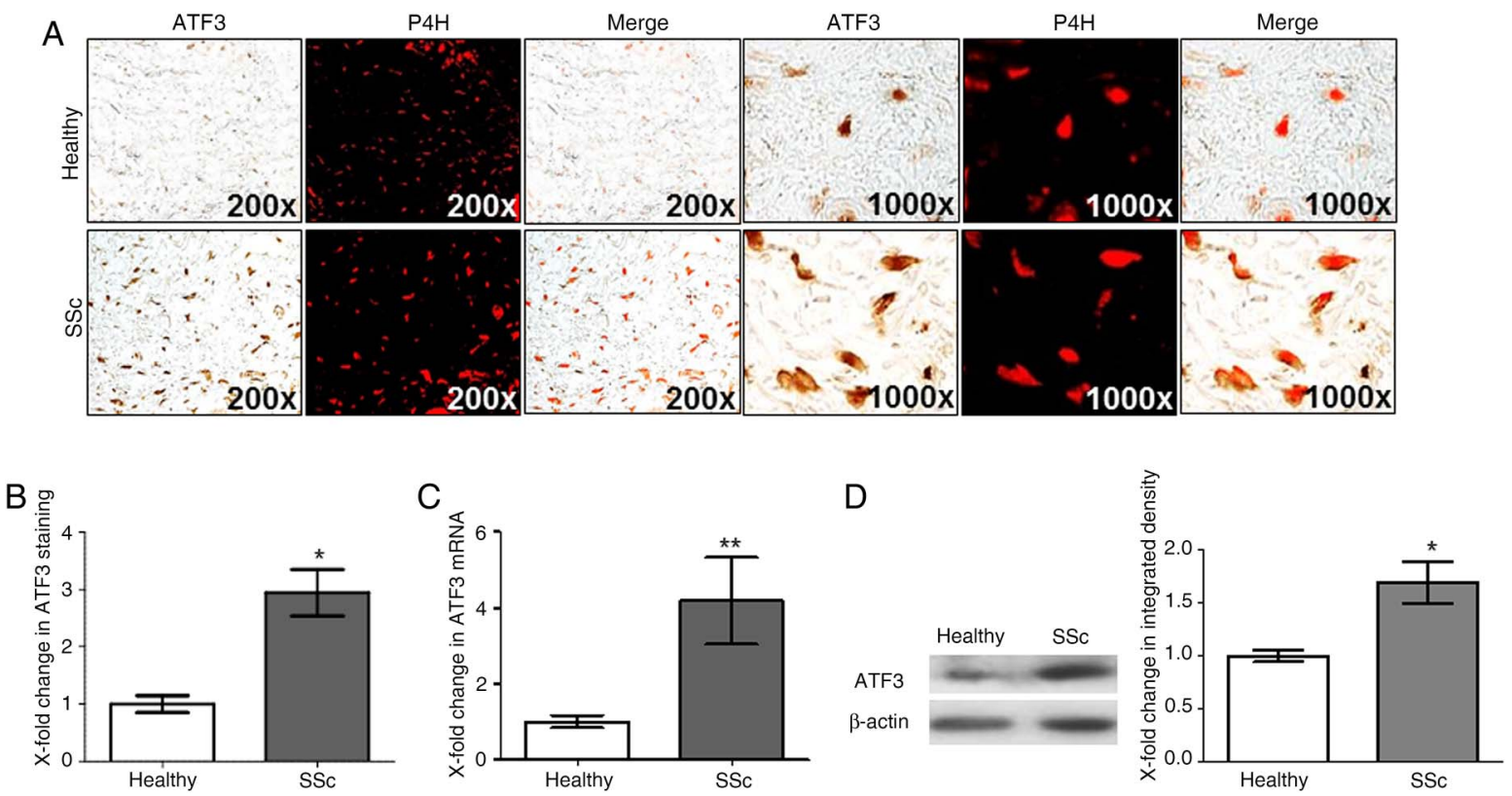

Figure 1 Increased expression of activating transcription factor 3 (ATF3) in skin from patients with systemic sclerosis (SSc). (A) Immunohistochemistry of ATF3 in SSc skin and matched healthy controls $(n=5)$. Double staining for ATF3 and fibroblast marker, prolyl-4-hydroxylase $\beta(P 4 H)$, on human skin. (B) Semiquantitative analyses confirmed the upregulation of ATF3 protein in fibroblasts in SSC skin ( $n=5)$. The upregulation of ATF3 persists in cultured SSc fibroblasts as indicated by mRNA $(n=5)(C)$ and protein levels $(n=5)(D)$ measured by reverse transcription quantitative PCR and western blot, respectively. Western blot signals were semiquantified by density analysis. ${ }^{*} p<0.05 ;{ }^{* *} p<0.01 ;{ }^{* * *} p<0.001$.

\section{ATF3 upregulates Smad signalling and fibroblast activation in a c-Jun-dependent manner}

To investigate the functional effects of ATF3 on fibroblasts, we first ectopically expressed ATF3 in healthy dermal fibroblasts. Exogenous expression of ATF3 by pCMV-ATF3 fostered canonical TGF $\beta$ signalling with increased Smad/CAGA reporter activation (figure $3 \mathrm{~A}$ ) and increased expression of plasminogen activator inhibitor type 1 (PAI-1), a classic target gene of TGF $\beta /$ Smad signalling (figure 3B). Fibroblasts expressing ATF3 also produced more collagen in response to TGF $\beta$ with higher mRNA levels of Col1a1 and increased collagen in the supernatant compared with fibroblasts transfected with the control plasmid (figure 3C).

In a complementary approach, we used fibroblasts lacking ATF3. Dermal fibroblasts isolated from ATF3 knockout (KO) mice were less responsive to TGF $\beta$ stimulation than the wildtype (WT) fibroblasts: they had reduced Smad/CAGA reporter activity, decreased mRNA levels of PAI-1, and decreased collagen in supernatant on stimulation with TGF $\beta$ (figure 3D-F). Together, these data identify ATF3 as a novel regulator of TGF $/$ Smad-dependent fibroblast activation.

Members of the activation protein 1 (AP-1) family of transcription factors have recently been identified as regulators of TGF $\beta$ signalling and key players in the pathogenesis of SSc. ${ }^{29-33}$ We thus hypothesise that ATF3 may regulate TGF $/$ Smad signalling in an AP-1-dependent manner. Indeed, pharmacological inhibition of AP-1 signalling by T-5224 completely abrogates the stimulatory effect of ATF3 on TGF $\beta$ signalling, as assayed by Col1a1 expression and soluble collagen (figure 4A). Moreover, CoIP studies demonstrated that TGF $\beta$ not only stimulated the recruitment of ATF3 to protein complexes containing Smad3, but also the recruitment of c-Jun (figure 4B), without affecting other members of the AP- 1 family such as JunD or Fra2 (data not shown). Immunoanalyses for ATF3 and c-Jun in SSc and healthy skin indicated costaining of ATF3 and
c-Jun in the same cells (figure 4C). Taken together, the CoIP and costaining data are consistent with the essential role of c-Jun for ATF3 (figure 4A).

\section{ATF3 deficiency reduces bleomycin-induced skin fibrosis in mice}

We next investigated the role of ATF3 in experimental models of fibrosis. We first examined the outcome of ATF3 KO mice in bleomycin-induced skin fibrosis. Injection of bleomycin induces localised inflammation and skin fibrosis, thereby resembling early, inflammatory stages of SSc. ${ }^{27} 34$ Although unchallenged ATF3 KO mice did not have an obvious skin phenotype, ATF3-deficient mice were strongly protected against experimental fibrosis (figure 5A). Bleomycin-induced dermal thickening, myofibroblast differentiation and hydroxyproline levels were all significantly reduced in ATF3 KO mice compared with WT littermates (figure $5 \mathrm{~B}-\mathrm{D}$ ). Consistent with impaired TGF $\beta$ signalling, ATF3 deficiency also prevented the upregulation of PAI-1 mRNA in bleomycin-challenged skin (figure 5E).

\section{ATF3 KO mice are protected from TBRlact-induced fibrosis}

We further evaluated the role of ATF3 using a mouse model of fibrosis induced by injecting the dermis with virus expressing a constitutively active TBRI. TBRIact-induced skin fibrosis was ameliorated in ATF3-deficient mice (figure 6A), with reduced dermal thickening, decreased myofibroblast counts and lower hydroxyproline levels compared with TBRIact WT mice (figure 6B-D). Overexpression of TBRIact also failed to increase the levels of PAI-1 mRNA in ATF3-deficient mice (figure 6E), further supporting the stimulatory effects of ATF3 on TGF 3 signalling.

\section{DISCUSSION}

The present study describes for the first time upregulation of ATF3 in fibroblasts in fibrotic conditions in the context of both human SSc and murine models of skin fibrosis. We demonstrate 


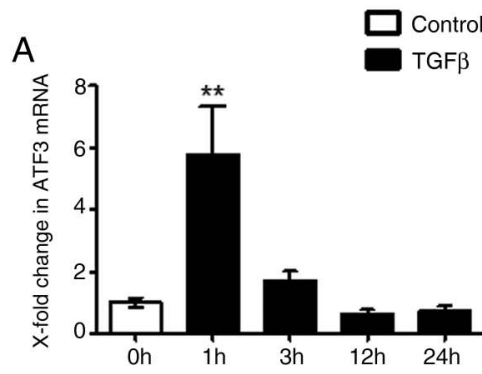

B

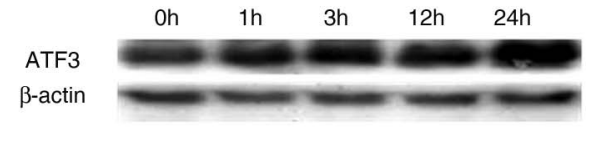

D

C
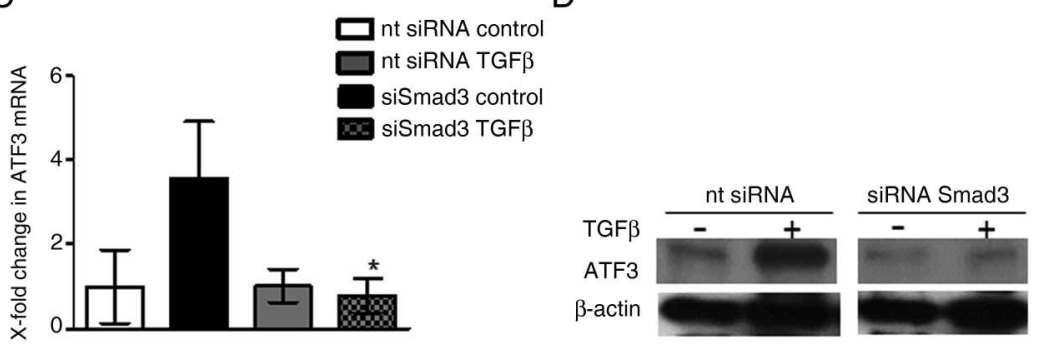

$\mathrm{E}$

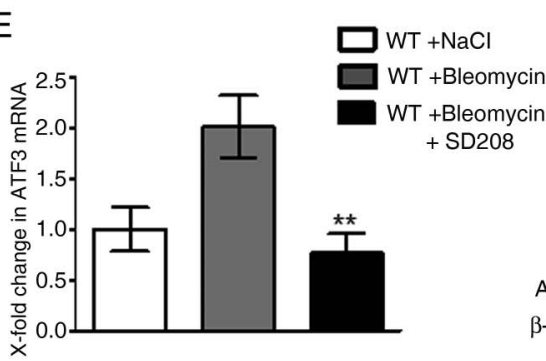

$\mathrm{F}$
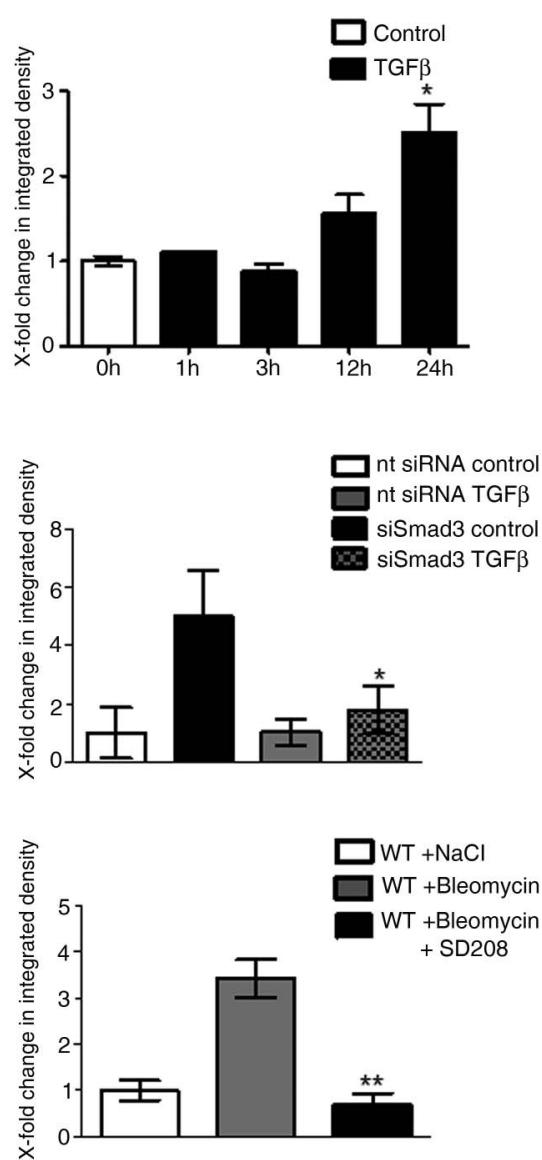

Figure 2 Activating transcription factor 3 (ATF3) is induced in canonical transforming growth factor- $\beta$ (TGF $\beta$ ) signalling. Increased mRNA (A) and protein (B) levels in fibroblasts on stimulation with TGF $\beta$ as measured by reverse transcription PCR and western blotting, respectively ( $n=5$ for both). Small interfering (si)RNA-mediated knockdown of Smad3 in fibroblasts prevents the TGF $\beta$-induced upregulation of ATF3 mRNA (C) and protein (D) $(n=5$ for both). Treatment with the selective TGF $\beta$ receptor type 1 kinase inhibitor, SD208, abrogates the increases in ATF3 mRNA (E) and protein (F) in the sum of bleomycin-challenged mice ( $n=5$ for both). nt, non-targeting; WT, wild-type. ${ }^{*} p<0.05$; ${ }^{* *} p<0.01 ;{ }^{* * *} p<0.001$.

on multiple experimental levels that TGF $\beta$ plays an important role in the induction of ATF3 in fibrotic conditions. (1) Incubation with recombinant TGF $\beta$ upregulated the mRNA and protein levels of ATF3 in cultured fibroblasts, but also in breast cancer cells. ${ }^{35}$ (2) Knockdown of Smad3 abrogated the stimulatory effects of TGF $\beta$ on ATF3 expression. (3) The expression of ATF3 was also upregulated in cultured SSc fibroblasts, which is consistent with endogenous activation of TGF $\beta$ signalling in SSc fibroblasts in vitro. ${ }^{36} 37$ (4) Treatment with SD208, a selective inhibitor of TBRI, ${ }^{28}$ completely prevented the upregulation of ATF3 in bleomycin-induced fibrosis. These findings identify TGF $\beta$ as an important regulator of ATF3. However, other factors such as hypoxia and reperfusion injury have also been shown to induce the expression of ATF3 and may also contribute to its overexpression in SSc. ${ }^{38} 39$

Furthermore, we characterise ATF3 as a crucial regulator of TGF $\beta$ signalling in SSc. ATF3-deficient fibroblasts are less responsive to TGF $\beta$, with impaired activation of Smad-dependent transcription in reporter assays and decreased levels of Smad target genes such as PAI-1 and type I collagens. In contrast, ectopic expression of ATF3 in fibroblasts potently enhances canonical TGF $\beta$ signalling and stimulates the expression of Smad target genes. We demonstrate that ATF3 interacts with Smad3 and that this interaction is induced by TGF $\beta$. Moreover, we demonstrate that the regulatory effects of ATF3 on TGF $\beta$ signalling are dependent on c-Jun, which is also recruited to the Smad3/ATF3 complex in a TGF $\beta$-dependent manner. Although further studies are required to determine whether interaction of ATF3 with Smad3 stimulates its DNA activity and enhances the recruitment of coactivators or fosters Smad-dependent transcription by other mechanisms, these findings highlight the stimulatory effects of ATF3 on canonical TGF $\beta$ signalling. Given that TGF $\beta$ induces the expression of ATF3, this stimulatory effect of ATF3 forms a positive feedback loop for TGF $\beta$. This feedback loop is not limited to fibroblasts, since it was also demonstrated in breast cancer epithelial cells. ${ }^{35}$ Thus, ATF3 is an endogenous enhancer of TGF $\beta$ signalling. This, combined with the induction of ATF3 by various stress signals, ${ }^{15}$ suggests that ATF3 may be a conduit for stress signals, to aberrantly activate TGF $\beta$ signalling and thus contribute to the pathogenesis of diseases such as breast cancer ${ }^{35}$ and SSc.

Functionally, the stimulatory effect of ATF3 on canonical TGF $\beta$ signalling directly translates into activating effects on fibroblasts. Fibroblasts expressing ATF3 are more susceptible to the profibrotic effects of TGF $\beta$, whereas the stimulatory effects of TGF $\beta$ on soluble collagen are blunted in ATF3 KO fibroblasts in vitro. ATF3 deficiency also interfered with the profibrotic effects of TGF $\beta$ in vivo. ATF3 KO mice were protected from experimental fibrosis induced by overexpression of a constitutively active TBRI and demonstrated reduced skin thickening, impaired differentiation of resting fibroblasts into myofibroblasts and decreased accumulation of collagen compared with WT 

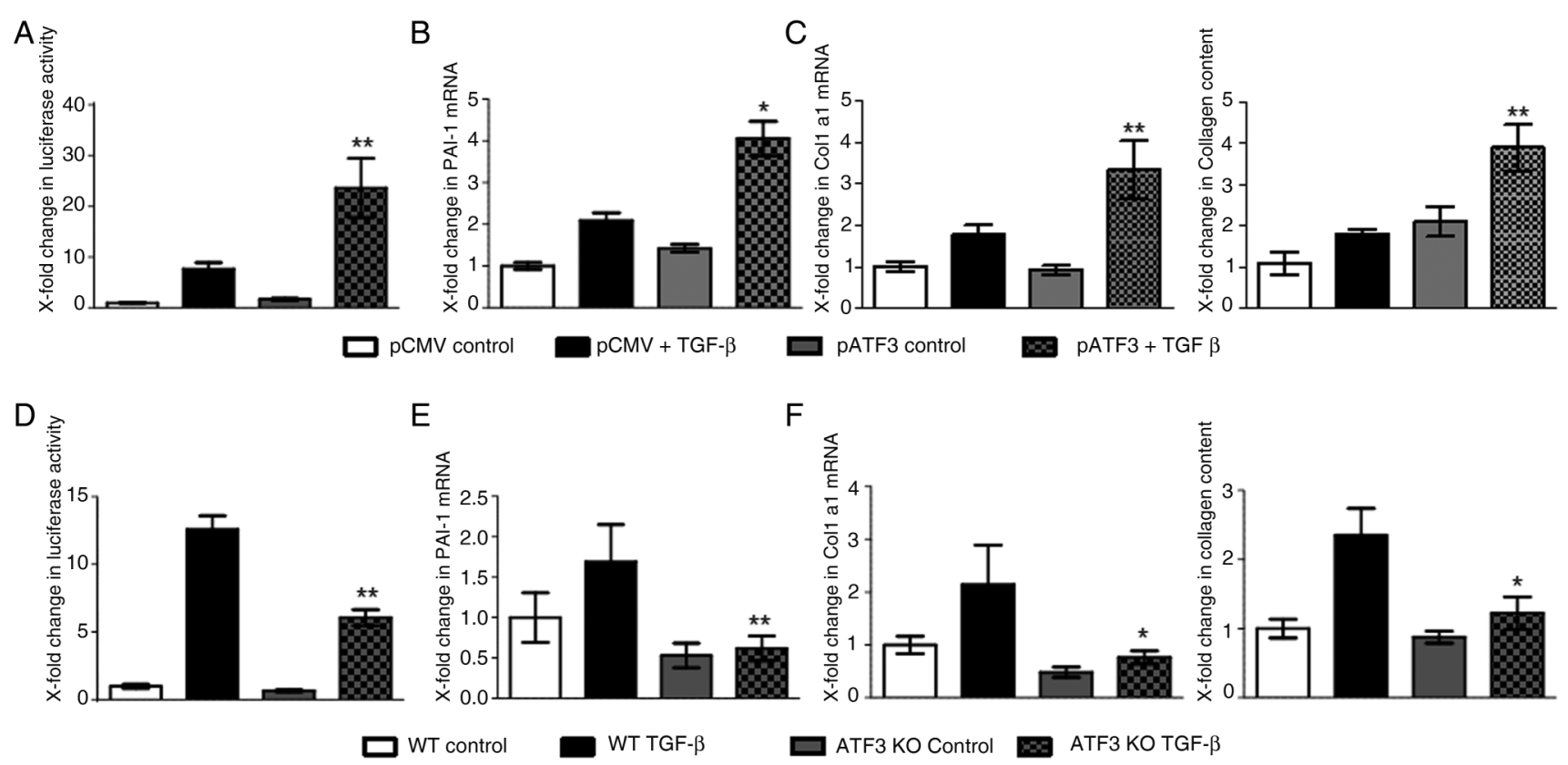

Figure 3 Activating transcription factor 3 (ATF3) regulates transforming growth factor- $\beta$ (TGF $\beta$ )-induced fibroblast activation. Ectopic expression of ATF3 in dermal fibroblasts from healthy human fibroblasts promotes TGF $\beta$-induced Smad-dependent transcription in reporter assays (A), increases the mRNA levels of the TGF $\beta / S m a d$ target genes, PAI-1 (B) and Col1a1 (C), and increases soluble collagen in the supernatant (C). ATF3 knockout (KO) dermal fibroblasts are less responsive to TGF $\beta$ as indicated by decreased Smad-reporter activity (D), impaired upregulation of plasminogen activator inhibitor type 1 (PAI-1) mRNA (E) and reduced soluble collagen (F), compared with wild-type (WT) fibroblasts. $n \geq 6$ for all experiments. ${ }^{*} p<0.05 ;{ }^{* *} p<0.01 ;{ }^{* * *} p<0.001$.

littermates. ATF3 KO mice were also protected from bleomycin-induced skin fibrosis, thereby confirming the central regulatory function of ATF3 in fibroblast activation in a multifactorial model of fibrosis. TGF $\beta$ receptor- and bleomycin-induced fibrosis resembles different aspects of SSc. The mouse model of bleomycin-induced dermal fibrosis mimics inflammatory stages of $\mathrm{SSc}$, in which fibroblasts are predominantly activated by profibrotic mediators released from infiltrating leucocytes. In contrast, the
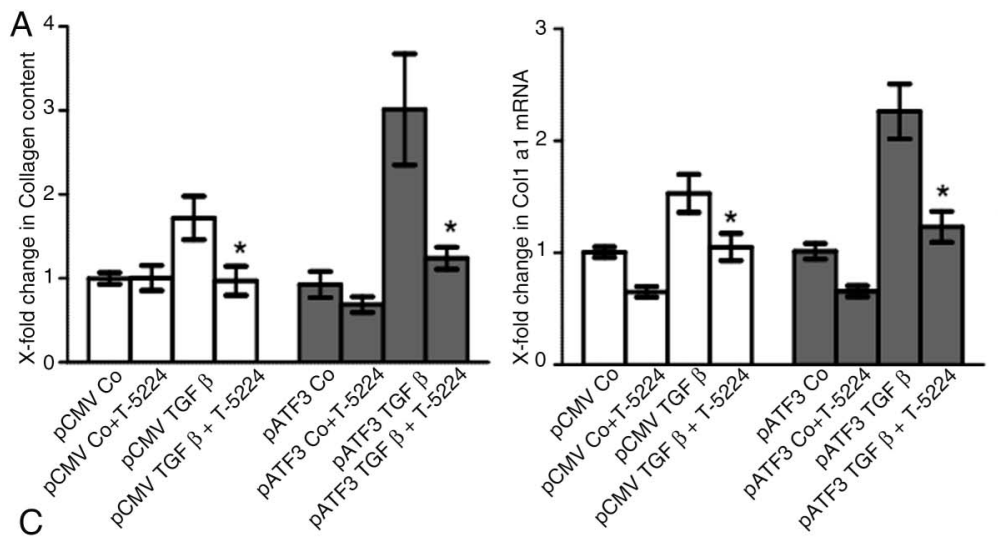

B

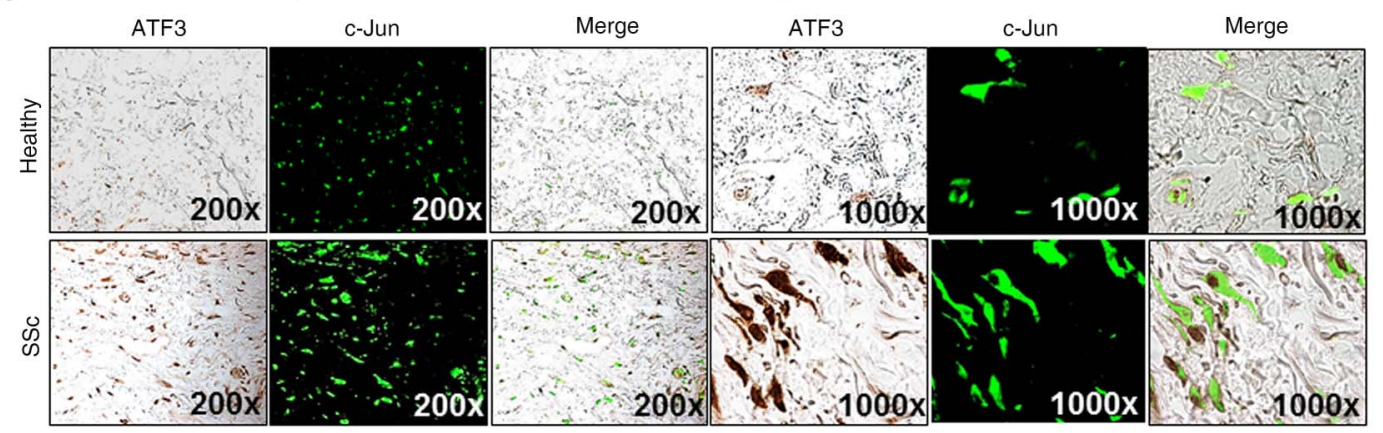

Figure 4 Activating transcription factor 3 (ATF3) regulates transforming growth factor- $\beta$ (TGF $\beta$ )/Smad signalling in a c-Jun-dependent manner. (A) Inhibition of activation protein 1 (AP-1) signalling by T-5224 abrogates the stimulatory effects of ATF3 ectopic expression on TGF $\beta$ signalling, as assayed by Col1a1 expression and soluble collagen $(n=5)$. (B) Coimmunoprecipitation demonstrating recruitment of ATF3 and c-Jun to protein complexes containing Smad3 on TGF $\beta$ stimulation $(n=5)$. (C) Immunohistochemistry (for ATF3) and immunofluorescence (for c-Jun) analyses demonstrating colocalisation of ATF3 and c-Jun in systemic sclerosis (SSc) and healthy skin ( $n=5)$. ${ }^{*} p<0.05 ;{ }^{* *} p<0.01 ;{ }^{* * *} p<0.001$. 


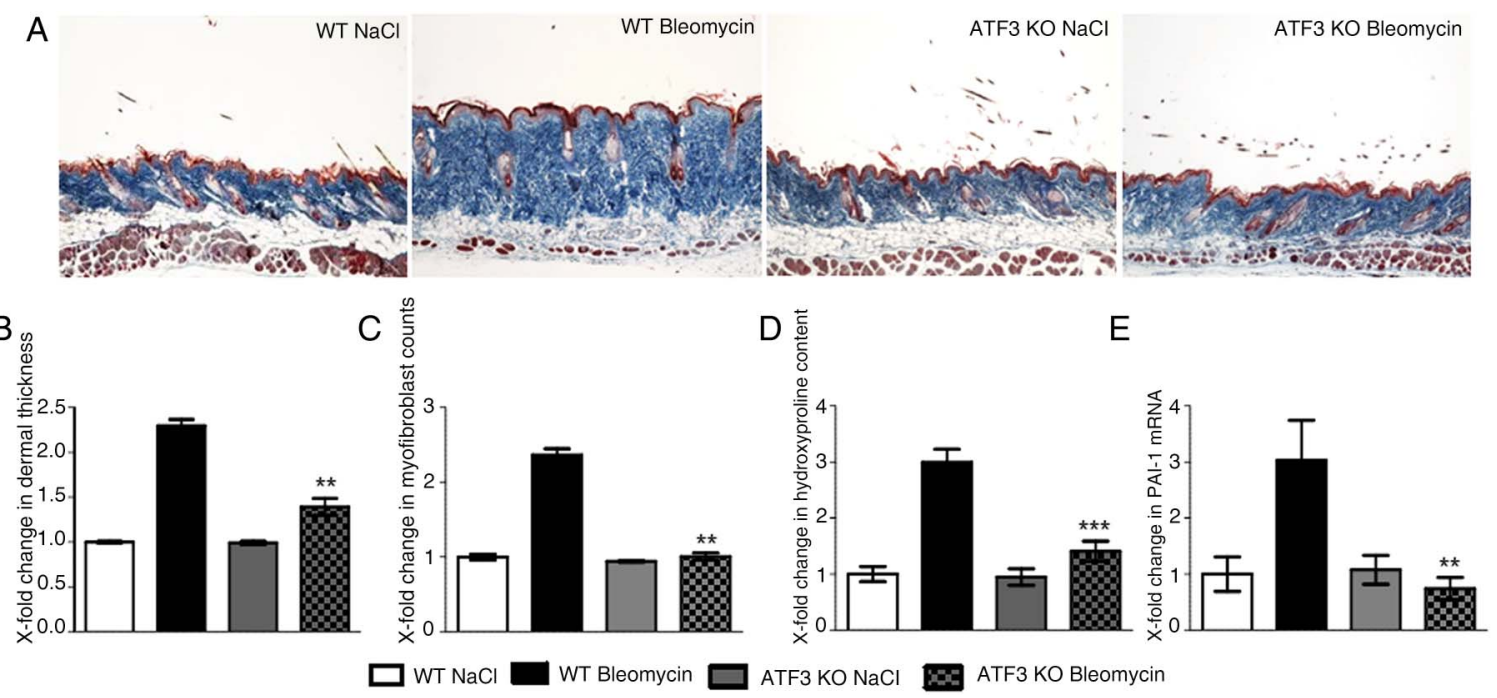

Figure 5 Mice lacking activating transcription factor 3 (ATF3) are protected from bleomycin-induced skin fibrosis. (A) Representative images of Masson trichrome-stained skin are shown at 100-fold magnification. Scale bar, $100 \mu \mathrm{m}$. (B) Dermal thickness. (C) $\alpha$-Smooth muscle actin-positive myofibroblasts. (D) Hydroxyproline content. (E) mRNA levels of transforming growth factor- $\beta$ (TGF $\beta$ ) target gene, plasminogen activator inhibitor type 1 (PAl-1). All groups consisted of $\geq 6$ mice each. ${ }^{*} p<0.05 ;{ }^{* *} p<0.01 ;{ }^{* *} p<0.001$.

mouse model of TGF $\beta$ receptor-induced fibrosis resembles patients with SSc in which inflammatory infiltrates have largely resolved and in which fibroblasts are endogenously activated because of persistent TGF $\beta$ signalling. ${ }^{29}{ }^{39}$ Since ATF3 deficiency ameliorated fibrosis in both models, it indicates that targeting ATF3 may exert antifibrotic effects in inflammatory as well as non-inflammatory subsets of patients with SSc. However, considering the complex pathogenesis of SSc and its systemic involvement, further in vivo studies are required to further characterise the role of ATF3 in the pathogenesis of SSc. We focused on the role of ATF3 in skin fibrosis as the most commonly affected tissue. Further studies should also analyse commonly affected internal organs such as lungs, gastrointestinal tract and heart. Moreover, we focused on the role of ATF3 in the pathogenesis of fibrosis. However, the expression of ATF3 in the vascular wall demonstrated by immunohistochemistry in our study (data not shown) may indicate that ATF3 also contributes to the vascular pathogenesis of SSc. This hypothesis should be further evaluated in murine models of SSc-associated vasculopathy such as Fra-2 transgenic mice or urokinase-type plasminogen activator receptor KO mice. $^{3140}$

In summary, our study characterises ATF3 as an amplifier of TGF $\beta$ signalling in SSc fibroblasts. ATF3 is induced by TGF $\beta$ in a Smad3-dependent manner and in turn enhances the profibrotic effects of TGF $\beta$ signalling in a c-Jun-dependent manner. Knockout of ATF3 impairs TGF $\beta$-induced transcription of Smad target genes and fibroblast activation, and ameliorates experimental fibrosis. These findings thus identify ATF3 as a novel regulator of fibroblast activation in fibrotic diseases such as SSc.

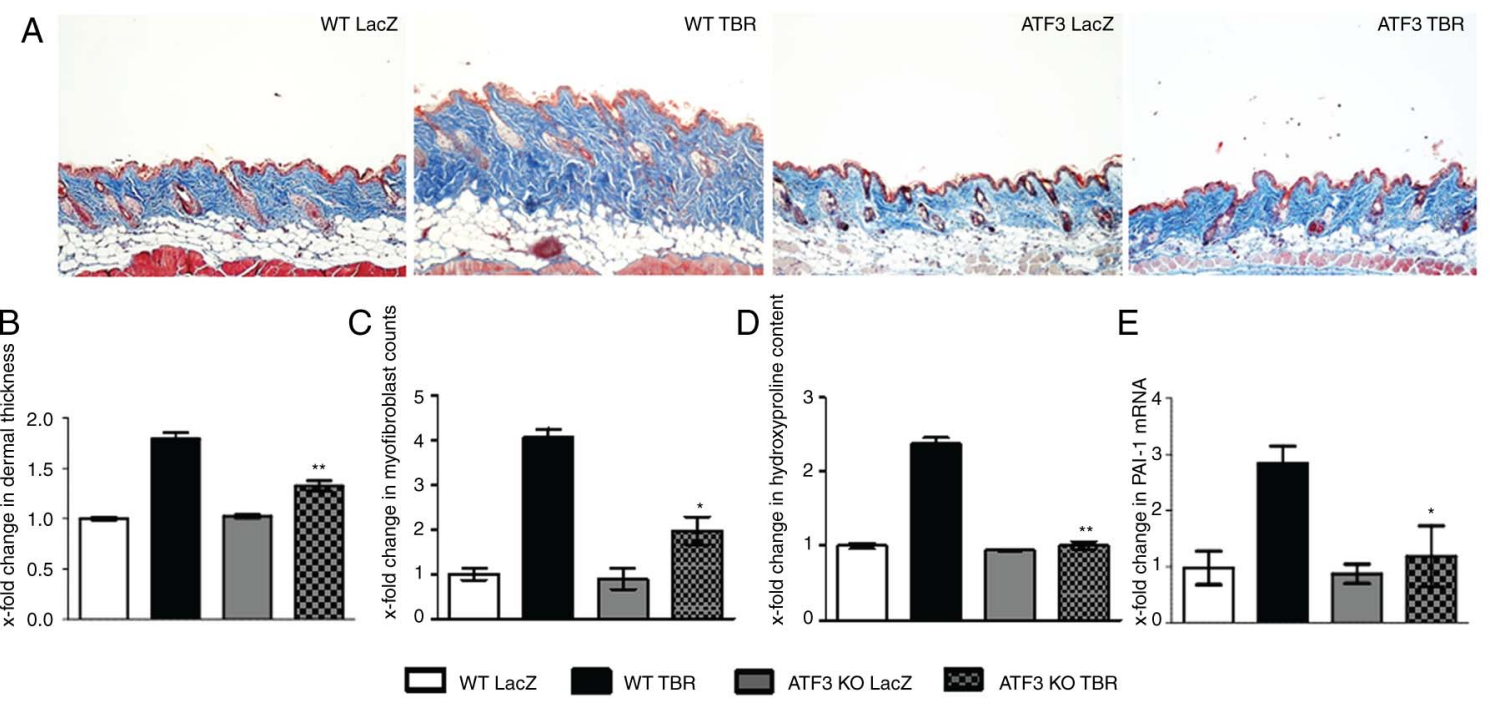

Figure 6 Mice lacking activating transcription factor 3 (ATF3) are protected from type $V$ adenovirus coding for TBRI (adTBRI)-induced fibrosis. (A) Representative images of Masson trichrome-stained skin are shown at 100-fold magnification. Scale bar, $100 \mu \mathrm{m}$. (B) Dermal thickness. (C) $\alpha$-Smooth muscle actin-positive myofibroblasts. (D) Hydroxyproline content. (E) mRNA levels of transforming growth factor- $\beta$ (TGF $\beta$ ) target gene, plasminogen activator inhibitor type 1 (PAl-1). All groups consisted of $\geq 6$ mice each. ${ }^{*} p<0.05 ;{ }^{* *} p<0.01 ;{ }^{* * *} p<0.001$. 
Acknowledgements We thank Katja Dreißigacker, Regina Kleinlein, Rosella Mancuso and Monica Pascual Mate for excellent technical assistance.

Contributors Design of the study: TM and JHWD. Acquisition of data: TM, KP-Z, $P Z, A R, B Z, C B, C D$ and JH. Interpretation of data: $T M, K P-Z, P Z, A R, C B, C D, H T$, OD, GS and JHWD. Manuscript preparation: TM and JHWD.

Funding Grants DI 1537/4-1, DI 1537/5-1, DI 1537/7-1, DI 1537/8-1, DI 1537/ 9-1, BE 5191/1-1, AK 144/1-1 and SCHE 1583/7-1 from the Deutsche Forschungsgemein-schaft, grants A57 and J29 from the IZKF in Erlangen and a Career Support Award of Medicine from the Ernst Jung Foundation.

Competing interests OD has consultancy relationships and/or has received research funding from Actelion, Pfizer, Ergonex, BMS, Sanofi-Aventis, United BioSource Corporation, medac, Biovitrium, Boehringer Ingelheim, Novartis, 4D Science and Active Biotec in the area of potential treatments of scleroderma; JHWD has consultancy relationships and/or has received research funding from Actelion, Pfizer, Ergonex, BMS, Celgene, Bayer Pharma, Boehringer Ingelheim, JB Therapeutics, Sanofi-Aventis, Novartis, Array Biopharma, KaroBio and Active Biotech in the area of potential treatments of scleroderma and is stock owner of $4 D$ Science $\mathrm{GmbH}$.

Ethics approval University of Erlangen-Nuremberg.

Provenance and peer review Not commissioned; externally peer reviewed.

Data sharing statement Raw data can be provided upon request.

\section{REFERENCES}

1 Gabrielli A, Avvedimento EV, Krieg T. Scleroderma. N Engl J Med 2009:360:1989-2003.

2 Akhmetshina A, Palumbo K, Dees C, et al. Activation of canonical Wnt signalling is required for TGF-beta-mediated fibrosis. Nat Commun 2012;3:735.

3 Hai T, Hartman MG. The molecular biology and nomenclature of the activating transcription factor/cAMP responsive element binding family of transcription factors: activating transcription factor proteins and homeostasis. Gene 2001;273:1-11.

4 Landschulz WH, Johnson PF, McKnight SL. The leucine zipper: a hypothetical structure common to a new class of DNA binding proteins. Science 1988;240:1759-64.

5 Chen BP, Liang G, Whelan J, et al. ATF3 and ATF3 delta Zip. Transcriptional repression versus activation by alternatively spliced isoforms. I Biol Chem 1994;269:15819-26.

6 Hai TW, Liu F, Coukos WJ, et al. Transcription factor ATF CDNA clones: an extensive family of leucine zipper proteins able to selectively form DNA-binding heterodimers. Genes Dev 1989;3:2083-90.

7 Hai T, Curran T. Cross-family dimerization of transcription factors Fos/Jun and ATF/ CREB alters DNA binding specificity. Proc Natl Acad Sci USA 1991;88:3720-4.

8 Hsu JC, Laz T, Mohn KL, et al. Identification of LRF-1, a leucine-zipper protein that is rapidly and highly induced in regenerating liver. Proc Natl Acad Sci USA 1991;88:3511-5.

9 Hsu JC, Bravo R, Taub R. Interactions among LRF-1, JunB, C-Jun, and C-Fos define a regulatory program in the $\mathrm{G} 1$ phase of liver regeneration. $\mathrm{Mol} \mathrm{Cell}$ Biol 1992;12:4654-65.

10 Chu HM, Tan Y, Kobierski LA, et al. Mol Endocrinol 1994;8:59-68.

11 Fawcett TW, Martindale JL, Guyton KZ, et al. Complexes containing activating transcription factor (ATF)/CAMP-responsive-element-binding protein (CREB) interact with the CCAAT/enhancer-binding protein (C/EBP)-ATF composite site to regulate Gadd153 expression during the stress response. Biochem J 1999;339(Pt 1):135-41.

12 Nilsson M, Toftgard R, Bohm S. Activated Ha-Ras but not TPA induces transcription through binding sites for activating transcription factor 3/Jun and a novel nuclear factor. J Biol Chem 1995;270:12210-8.

13 Hai T, Wolfgang CD, Marsee DK, et al. ATF3 and stress responses. Gene Expr 1999;7:321-35.

14 Bakin AV, Stourman NV, Sekhar KR, et al. Smad3-ATF3 signaling mediates TGF-beta suppression of genes encoding Phase II detoxifying proteins. Free Radic Biol Med 2005;38:375-87.

15 Kang Y, Chen CR, Massague J. A self-enabling TGFbeta response coupled to stress signaling: Smad engages stress response factor ATF3 for Id1 repression in epithelial cells. Mol Cell 2003;11:915-26.

16 Gabrielli A, Svegliati S, Moroncini G, et al. New insights into the role of oxidative stress in scleroderma fibrosis. Open Rheumatol J 2012;6:87-95.
17 van den Hoogen F, Khanna D, Fransen J, et al. 2013 classification criteria for systemic sclerosis: an American college of rheumatology/European league against rheumatism collaborative initiative. Ann Rheum Dis 2013;72:1747-55.

18 Beyer C, Zenzmaier C, Palumbo-Zerr K, et al. Stimulation of the soluble guanylate cyclase ( $\mathrm{sGC}$ ) inhibits fibrosis by blocking non-canonical TGFbeta signalling. Ann Rheum Dis 2015;74:1408-16.

19 Dees C, Schlottmann I, Funke R, et al. The Wnt antagonists DKK1 and SFRP1 are downregulated by promoter hypermethylation in systemic sclerosis. Ann Rheum Dis 2014;73:1232-9.

20 Zerr $\mathrm{P}$, Vollath $\mathrm{S}$, Palumbo-Zerr $\mathrm{K}$, et al. Vitamin D receptor regulates TGF-beta signalling in systemic sclerosis. Ann Rheum Dis 2015;74:e20.

21 Dees C, Akhmetshina A, Zerr P, et al. Platelet-derived serotonin links vascular disease and tissue fibrosis. J Exp Med 2011;208:961-72.

22 Dees C, Tomcik M, Zerr P, et al. Notch signalling regulates fibroblast activation and collagen release in systemic sclerosis. Ann Rheum Dis 2011;70:1304-10.

23 Tomcik M, Palumbo-Zerr K, Zerr P, et al. S100A4 amplifies TGF-beta-induced fibroblast activation in systemic sclerosis. Ann Rheum Dis 2014;73:1215-22.

24 Tomcik M, Zerr P, Pitkowski J, et al. Heat shock protein 90 (Hsp90) inhibition targets canonical TGF-beta signalling to prevent fibrosis. Ann Rheum Dis 2014;73:1215-22.

25 Dees C, Tomcik M, Palumbo-Zerr K, et al. JAK-2 as a novel mediator of the profibrotic effects of transforming growth factor beta in systemic sclerosis. Arthritis Rheum 2012;64:3006-15.

26 Beyer C, Reich N, Schindler SC, et al. Stimulation of soluble guanylate cyclase reduces experimental dermal fibrosis. Ann Rheum Dis 2012;71:1019-26.

27 Beyer C, Schramm A, Akhmetshina A, et al. beta-catenin is a central mediator of pro-fibrotic Wnt signaling in systemic sclerosis. Ann Rheum Di 2012;71: 761-7.

28 Uhl M, Aulwurm S, Wischhusen J, et al. SD-208, a novel transforming growth factor beta receptor I kinase inhibitor, inhibits growth and invasiveness and enhances immunogenicity of murine and human glioma cells in vitro and in vivo. Cancer research 2004;64:7954-61.

29 Maurer B, Busch N, Jungel A, et al. Transcription factor fos-related antigen-2 induces progressive peripheral vasculopathy in mice closely resembling human systemic sclerosis. Circulation 2009;120:2367-76.

30 Maurer B, Reich N, Juengel A, et al. Fra-2 transgenic mice as a novel model of pulmonary hypertension associated with systemic sclerosis. Ann Rheum Dis 2012;71:1382-7.

31 Maurer B, Distler JH, Distler O. The Fra-2 transgenic mouse model of systemic sclerosis. Vasc Pharmacol 2013;58:194-201.

32 Palumbo K, Zerr $\mathrm{P}$, Tomcik $\mathrm{M}$, et al. The transcription factor JunD mediates transforming growth factor \{beta\}-induced fibroblast activation and fibrosis in systemic sclerosis. Ann Rheum Dis 2011;70:1320-6.

33 Reich N, Maurer B, Akhmetshina A, et al. The transcription factor Fra-2 regulates the production of extracellular matrix in systemic sclerosis. Arthritis Rheum 2010;62:280-90.

34 Beyer C, Schett G, Distler O, et al. Animal models of systemic sclerosis: prospects and limitations. Arthritis Rheum 2010;62:2831-44.

35 Yin X, Wolford CC, Chang YS, et al. ATF3, an adaptive-response gene, enhances TGF\{beta\} signaling and cancer-initiating cell features in breast cancer cells. I Cell Sci 2010;123(Pt 20):3558-65.

36 LeRoy EC. Increased collagen synthesis by scleroderma skin fibroblasts in vitro: a possible defect in the regulation or activation of the scleroderma fibroblast. I Clin Invest 1974:54:880-9.

37 Yamamoto T, Takagawa S, Katayama I, et al. Animal model of sclerotic skin. I: Local injections of bleomycin induce sclerotic skin mimicking scleroderma. J Invest Dermatol 1999;112:456-62.

38 Distler JH, Jungel $\mathrm{A}$, Pileckyte $\mathrm{M}$, et al. Hypoxia-induced increase in the production of extracellular matrix proteins in systemic sclerosis. Arthritis Rheum 2007:56:4203-15.

39 Men G, Peyman GA, Genaidy M, et al. The role of recombinant lysine-plasminogen and recombinant urokinase and sulfur hexafluoride combination in inducing posterior vitreous detachment. Retina 2004;24:199-209.

40 Manetti M, Rosa I, Milia AF, et al. Inactivation of urokinase-type plasminogen activator receptor (UPAR) gene induces dermal and pulmonary fibrosis and peripheral microvasculopathy in mice: a new model of experimental scleroderma? Ann Rheum Dis 2014;73:1700-9. 


\section{$A$ Activating transcription factor 3 regulates canonical TGF $\beta$ signalling in systemic sclerosis}

Tatjana Mallano, Katrin Palumbo-Zerr, Pawel Zerr, Andreas Ramming, Barbara Zeller, Christian Beyer, Clara Dees, Jingang Huang, Tsonwin Hai, Oliver Distler, Georg Schett and Jörg H W Distler

Ann Rheum Dis2016 75: 586-592 originally published online January 14, 2015

doi: 10.1136/annrheumdis-2014-206214

Updated information and services can be found at:

http://ard.bmj.com/content/75/3/586

These include:

References This article cites 40 articles, 24 of which you can access for free at: http://ard.bmj.com/content/75/3/586\#ref-list-1

Email alerting service

Receive free email alerts when new articles cite this article. Sign up in the box at the top right corner of the online article.

Topic Articles on similar topics can be found in the following collections

Collections

Immunology (including allergy) (5144)

Connective tissue disease (4253)

\section{Notes}

To request permissions go to:

http://group.bmj.com/group/rights-licensing/permissions

To order reprints go to:

http://journals.bmj.com/cgi/reprintform

To subscribe to BMJ go to:

http://group.bmj.com/subscribe/ 